\title{
Study of Band-Edge Modes in GaN-Based Photonic Crystal Surface-Emitting Lasers by the Multiple-Scattering Method
}

\author{
Peng-Hsiang Weng, Tzeng-Tsong Wu, and Tien-Chang Lu, Member, IEEE
}

\begin{abstract}
In this paper, we have investigated the mode patterns in real space and reciprocal space, and threshold gain at different band edges in GaN-based photonic crystal surface-emitting lasers (PCSELs) by using the multiple-scattering method. The characteristics at each band edge ( $\Gamma 1, \mathrm{~K} 2$, and M3) of PCSELs are simulated and discussed. In addition, GaN-based PCSELs operated at different band edges have been fabricated and measured. The experimental results of threshold show good agreement with the simulation results.
\end{abstract}

Index Terms-Distributed feedback (DFB), GaN, multiple scattering, photonic crystal, surface-emitting lasers (SELs).

\section{INTRODUCTION}

I $\mathrm{N}$ RECENT years, the 2-D photonic crystal employing the 2-D distributed feedback (DFB) mechanism has been widely utilized in optoelectronic devices, such as photonic crystal surface-emitting lasers (PCSELs) and LEDs) [1]-[4]. With the careful selection of normalized frequency at the photonic band edges by adjusting emission wavelength and photonic crystal lattice constant, specific Bragg diffraction will occur not only to realize the stimulated resonant condition in the inplane direction but also to achieve the laser output coupling in the surface emission direction. Such a mechanism is advantageous because PCSELs could lase over a broad area defined by the photonic crystal patterns, which in turn implies potential to achieve high-output power emission, and operate in single-mode emission with a low divergence angle. Based on these characteristics, PCSELs could be excellent candidates for many applications including high-definition laser printers, highdensity optical data storage systems and microlaser/picolaser projectors. So far, many investigations on PCSELs have been explored and the emission wavelength of these devices covers from short wavelength to terahertz regions [5]-[9]. However, the 3-D features inherited in the PCSELs pose great challenges to build a mature analysis and calculation tool to design the

Manuscript received June 20, 2011; revised August 20, 2011; accepted September 5, 2011. The work was supported by the Ministry of Education ATU program, and in part by the National Science Council of Taiwan under Contract NSC 98-3114-M-009-001.

The authors are with the Department of Photonics and Institute of Electro-Optical Engineering, National Chiao Tung University, Hsinchu 30050, Taiwan (e-mail: dopin314@gmail.com; mulderbob.eo98g@nctu.edu.tw; timtclu@mail.nctu.edu.tw).

Color versions of one or more of the figures in this paper are available online at http://ieeexplore.ieee.org.

Digital Object Identifier 10.1109/JSTQE.2011.2169237 laser structure. There have been several researchers focusing on the theoretical analysis of the DFB mechanism on PCSELs by using different calculation methods. The plane-wave expansion method (PWEM) to calculate the lasing threshold by analyzing the group-velocity anomaly in the 2-D photonic crystal was presented in [10]. The authors used the coupled wave theory to explain the threshold gains and deviations from the Bragg frequency for square and triangular lattices of PCSELs in [11] and [12]. The finite-difference time-domain (FDTD) method was applied to comprehend the quality factor for near bandedge modes in the finite-size 2-D photonic crystals in [13]. The multiple-scattering method (MSM) to evaluate laser characteristics in photonic crystal circular rods with optical gains was reported in [14] and [15]. The authors also manipulated the coupled wave theory to shed light on the coupling coefficients of different band edges in PCSELs with a triangular lattice [16]. Although the aforementioned different theoretical methods take advantageous positions in some respects, while analyzing certain laser characteristics, each of them suffers limitations in calculation of the PCSEL structures. The coupled wave theory can provide a quick estimation of threshold conditions in PCSELs but the boundary condition needs to be carefully dealt with and the analysis of arbitrary defect or lattice patterns requires further development. As for the FDTD method, it consumes numerous computer memories and calculation time to simulate the finite PC structure. In spite of the 3-D calculation capability provided by the FDTD, it is a complex way to calculate the threshold gain from the FDTD. On the other hand, the 2-D PWEM applies only to the infinite photonic crystal patterns instead of the real device such that the boundary conditions of the PCSELs are not taken into account. Finally, the MSM shows good capability, such as faster calculation time, considering gain coefficients within photonic crystal materials with arbitrary defect or lattice patterns.

Therefore, the purpose of this paper is to investigate the characteristics of different band-edge modes including $\Gamma 1, \mathrm{~K} 2$, and M3 (defined and shown in Fig. 3) in GaN-based PCSELs with a triangular lattice pattern. The mode patterns in real space, reciprocal space, and threshold gain of different band-edge modes in GaN-based PCSELs were calculated by using the MSM. The paper is outlined as follows. The model of the MSM is briefly introduced and derived in Section II. Then, the fabrication process of three kinds of GaN-based PCSELs operated at different bad edges and the experimental setups are presented in Section III. In Section IV, different characteristics, such as mode patterns in real space, threshold gains, and mode patterns in reciprocal 


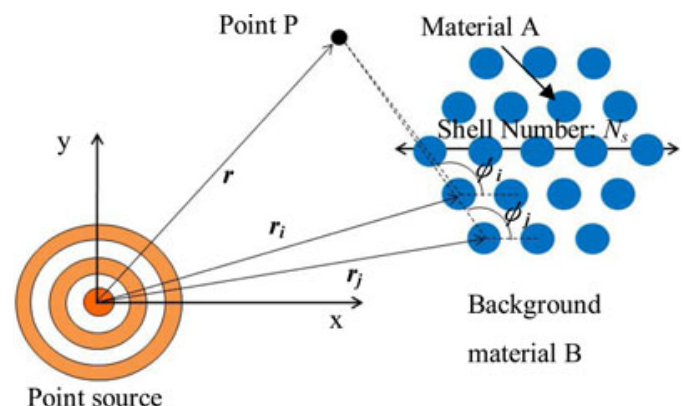

Fig. 1. Representation of the field at point $P$ contributed by scattered fields from various circular photonic structures and a point source used in the MSM.

space at $\Gamma 1, \mathrm{~K} 2$, and M3 band edges, are investigated and discussed. The threshold power density of GaN-based PCSELs at $\Gamma 1, \mathrm{~K} 2$, and $\mathrm{M} 3$ band edges are presented. The simulation results of the threshold gain for each band-edge modes are matched well to the experimental results. Finally, a summary of these results and prospects is given in Section $\mathrm{V}$.

\section{MultiPle-ScAtTering Method}

In our calculation model, the 2-D photonic crystal basis consisting of cylinder arrays is placed in a uniform gain medium. As shown in Fig. 1, the background material B is an isotropic gain medium, which is characterized by a complex dielectric constant, and circular rods are made up of material A. The dielectric constant of material B can be written as follows:

$$
\varepsilon_{B}(\omega)=\varepsilon_{b}(\omega)-i \frac{2 c \sqrt{\varepsilon_{b}}}{\omega} k_{b}^{\prime \prime} .
$$

The constant $\varepsilon_{b}$ represents the dielectric constant varied with the frequency of light, and the $k_{b}^{\prime \prime}$ represents the amplitude gain coefficient of material B (background wave number of background material $\mathrm{B}$, i.e., $\left.k_{B}=k_{b}-i k_{b}^{\prime \prime}\right)$. A point source at the origin point emits a monochromatic wave to cylinders with the finite number, locating at $r_{i}(i=1,2, \ldots, N)$. The field scattered from each cylinder shall response to the total incident field composed of the direct field from the source and the multiple scattered fields from other cylinders. The scattered field from the $j$ th cylinder can be written as

$$
\Phi_{s}\left(\vec{r}, \vec{r}_{j}\right)=\sum_{n=-\infty}^{\infty} i \pi A_{n}^{j} H_{n}^{(1)}\left(k_{B}\left|\vec{r}-\vec{r}_{j}\right|\right) e^{i n \phi_{\vec{r}-\vec{r}_{j}}}
$$

where $H_{n}^{(1)}$ is the $n$ th-order Hankel function of the first kind, $k$ is the wave number, $\psi$ is the azimuthal angle, and $A_{n}^{j}$ is the $n$th expansion Bessel coefficient of the $j$ th cylinder. Similarly, the total incident field around the $i$ th cylinder $(i=1,2, \ldots, N ; i \neq j)$ can be expressed as in the Bessel function form:

$$
\Phi_{\text {in }}^{i}(\vec{r})=\sum_{n=-\infty}^{\infty} B_{n}^{i} J_{n}\left(k_{B}\left|\vec{r}-\vec{r}_{i}\right|\right) e^{i n \phi \vec{r}^{-} \vec{r}_{i}} .
$$

The coefficients $A_{n}^{i}$ and $B_{n}^{i}$ can be solved by expressing the scattered field $\Phi_{s}\left(\vec{r}, \vec{r}_{j}\right)$, for each $i \neq j$, in terms of the modes with respect to the $i$ th cylinder by the addition theorem of the Bessel function [17]. The final field reaching a receiver located at $\vec{r}$ is the sum of direct wave from the source and the scattered fields from all the cylinders. Therefore, the external field of cylinders could be expressed as

$$
\begin{aligned}
\Phi_{\mathrm{ext}}^{i}(\vec{r})=\sum_{n=-\infty}^{\infty} & {\left[B_{n}^{j} J_{n}\left(k_{B}\left|\vec{r}-\vec{r}_{i}\right|\right)\right.} \\
& \left.+i \pi A_{n}^{j} H_{n}^{(1)}\left(k_{B}\left|\vec{r}-\vec{r}_{i}\right|\right)\right] e^{i n \phi \vec{r}_{r}-\vec{r}_{i}} .
\end{aligned}
$$

The aforementioned equation expresses a sum of external field from the incident field and the scattered field outside the $i$ th cylinder. In addition, we assume that the wave inside the $i$ th cylinder can be defined as

$$
\Phi_{\text {int }}^{i}(\vec{r})=\sum_{n=-\infty}^{\infty} D_{n}^{j} J_{n}\left(k_{A}\left|\vec{r}-\vec{r}_{i}\right|\right) e^{i n \phi \vec{r}_{r}-\vec{r}_{i}} .
$$

Here, $D_{n}^{i}$ is the assumption coefficient of the internal field. Then, the usual boundary conditions are considered. The field and the derivative field should be continuous across the interface between the cylinder and the surrounding material. We derive the correlation between $A_{n}^{i}$ and $B_{n}^{i}$ that leads to

$$
B_{n}^{i}=i \pi \Gamma_{n}^{i} A_{n}^{i}
$$

$\Gamma_{n}^{i}=\frac{H_{n}^{(1)}\left(k_{B} a^{i}\right) J_{n}^{\prime}\left(k_{A} a^{i}\right)-g\left(k_{B} / k_{A}\right) H_{n}^{(1)^{\prime}}\left(k_{B} a^{i}\right) J_{n}\left(k_{A} a^{i}\right)}{g\left(k_{B} / k_{A}\right) J_{n}^{\prime}\left(k_{B} a^{i}\right) J_{n}\left(k_{A} a^{i}\right)-J_{n}\left(k_{B} a^{i}\right) J_{n}^{\prime}\left(k_{A} a^{i}\right)}$

where $a^{i}$ is the radius of $i$ th cylinder, and $g$ is the dielectric constant ratio between materials $\mathrm{A}$ and $\mathrm{B}$. Then, the total system matrix of the photonic crystal can be obtained as follows:

$$
\Gamma_{n}^{i} A_{n}^{i}-\sum_{j=1, j \neq i}^{N} \sum_{l=-\infty}^{\infty} G_{l, n}^{i, j} A_{l}^{j}=T_{n}^{i}
$$

Here, we define

$$
T_{n}^{i}(\vec{r})=H_{-n}^{(1)}\left(k_{B}\left|\vec{r}_{i}\right|\right) e^{-i n \phi_{r_{i}}}
$$

and

$$
G_{l, n}^{i, j}=H_{l-n}^{(1)}\left(k_{B}\left|\vec{r}_{i}-\vec{r}_{j}\right|\right) e^{-i n \phi_{r_{i}-\vec{r}_{j}}}, \quad i \neq j .
$$

Here, $A_{n}^{i}$ and $T_{n}^{i}$ represent the expansion coefficients of scattering field and expansion coefficient of the incident field. Equation (8) can be simplified to an eigen-value problem: $M A=T$. The laser oscillation condition would be thus achieved under the condition that the value of vector $A$ / $T$ is divergent. Therefore, $\operatorname{det}(M)=0$ is the complex determinant equation used to search for a pair of variables of the threshold amplitude gain $k_{\mathrm{am}}^{\prime \prime}$ and the normalized frequency from $k=\omega / c$. Once the coefficient $A_{n}^{i}$ is determined, the total field outside of the cylinder at any spatial point is given by

$$
\begin{aligned}
& \Phi_{\text {ext }}(\vec{r}) \\
& \quad=i \pi H_{0}^{(1)}+\sum_{i=1}^{N} \sum_{n=-\infty}^{\infty} i \pi A_{n}^{i} H_{n}^{i}\left(k_{B}\left|\vec{r}-\vec{r}_{i}\right|\right) e^{i n \phi \vec{r}_{r}-\vec{r}_{i}} .
\end{aligned}
$$


We, then, solve the relation between $D_{n}^{i}$ and $A_{n}^{i}$ by the boundary condition and obtain $D_{n}^{i}=i \pi Z_{n}^{i} A_{n}^{i}$, where $Z_{n}^{i}$ is expressed as

$$
Z_{n}^{i}=\frac{-2 i /\left(\pi k_{B} a^{i}\right)}{J_{n}\left(k_{A} a^{i}\right) J_{n}^{\prime}\left(k_{B} a^{i}\right)-\left(k_{A} /\left(g k_{B}\right)\right) J_{n}\left(k_{B} a^{i}\right) J_{n}^{\prime}\left(k_{A} a^{i}\right)} .
$$

Therefore, the internal field of the $i$ th cylinder can be derived from (5) by the aforementioned correlation.

After pairs of the resonant-mode frequency and threshold amplitude gain are determined, the corresponding laser oscillation modes are investigated in terms of the magnetic-field distribution in the reciprocal space (or called $k$-space). Since the resonant mode will be diffracted to the photonic band edge, the field pattern in the reciprocal space is useful to clarify the resonant modes at different band edges. The field pattern in the reciprocal space can be calculated as

$$
\Phi(\vec{g})=\int_{|\vec{r}|<\infty} \Phi(\vec{r}) e^{-i \vec{g} \cdot \vec{r}} d \vec{r}
$$

where $\vec{g}$ is the 2-D reciprocal lattice vector. By observing the intensity distribution in the reciprocal space, the directions of the dominant waves can be determined and manifest the characteristics in the PCSELs.

\section{Device Structure AND MEasurement System}

In the experiment, the GaN-based PCSEL structure was grown on a $c$-plane 2-in sapphire by a metal-organic chemical vapor deposition (MOCVD) system. The epitaxial structure composed of a 2- $\mu$ m-thick GaN buffer layer, a 35-pair GaN/AlN distributed Bragg reflector (DBR), a 560-nm-thick n-GaN layer, ten pairs of InGaN/GaN multiple quantum wells (MQW), a 24$\mathrm{nm}$-thick AlGaN electron blocking layer, and a 175 -nm-thick p-GaN layer. The detailed growth parameters were reported in [6]. Then, the electron-beam lithography (EBL) was utilized to define the photonic crystal pattern with the triangular lattice on the sample surface. The photonic crystal pattern with the lattice constant $a$ was defined ranging from 180 to $300 \mathrm{~nm}$ to match different normalized frequency conditions and the circular hole diameter $r$ was chosen such that $r / a$ ranged from 0.18 to 0.3 . Here, the lattice constants are designed to make sure that the gain region would cover the lasing wavelength for particular band-edge points.

Then, the photonic crystal pattern was etched about $400 \mathrm{~nm}$ down to n-GaN by using the inductively coupled plasma system. The photonic crystal pattern was filled in a round area with a diameter of about $50 \mu \mathrm{m}$. The schematic cross section of the GaN-based PCSEL device structure is shown in Fig. 2(a). Fig. 2(b) shows the fundamental guided mode calculating by the transfer matrix method.

In the measurement system, a 355-nm $\mathrm{YVO}_{4}$ pulse laser was used as the excitation source in the micro-photoluminescence ( $\mu$-PL) system [6]. The pulse width is $0.5 \mathrm{~ns}$ and the repetition rate is $1 \mathrm{kHz}$. The pumping laser beam, with a spot size of $50 \mu \mathrm{m}$ in diameter, was normally incident onto the sample surface and covered the whole PC pattern area. A $15 \times$ objective lens with a numerical aperture of 0.32 was placed normally to the sample

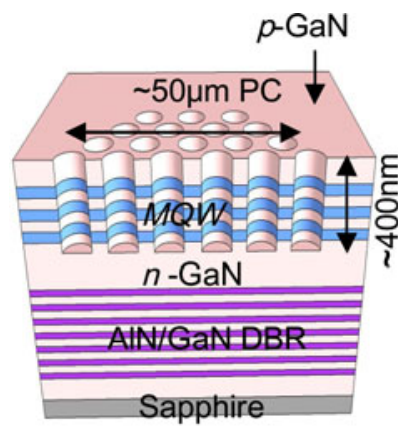

(a)

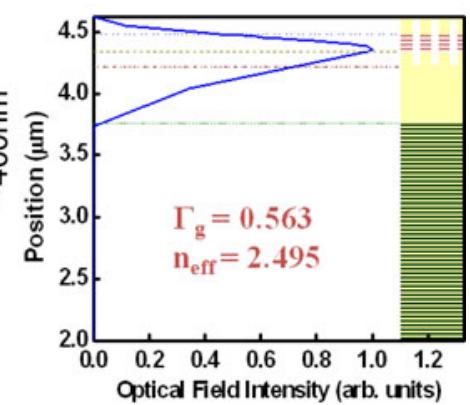

(b)
Fig. 2. (a) Schematics of the GaN-based PCSEL structures. (b) Fundamental guided mode calculated by the transfer matrix method.

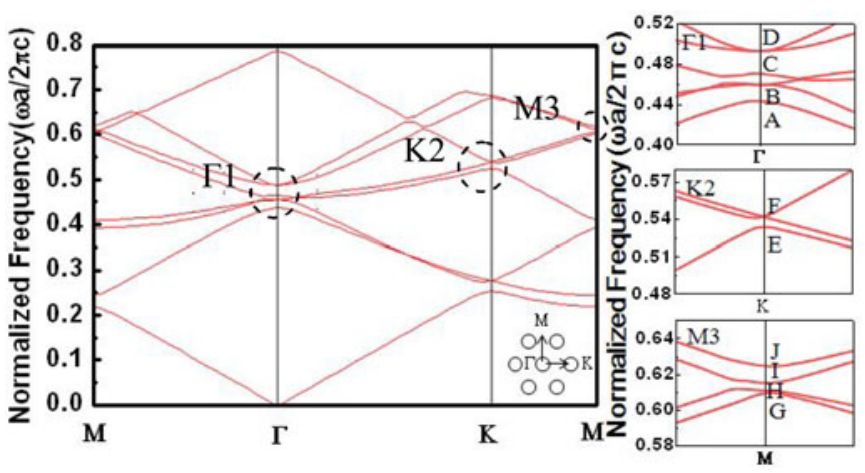

Fig. 3. Band structure for the photonic crystal with a triangular lattice in TE polarization. The dashed circles indicate the $\Gamma 1, \mathrm{~K} 2$, and $\mathrm{M} 3$ band edges, respectively. The inset shows the $\Gamma, \mathrm{K}$, and $\mathrm{M}$ directions in the real space. (Right) Detailed band structures near each band edge.

to collect the light emission from the top of the photonic crystal pattern. The light was, then, collected through a fiber with a $600 \mu \mathrm{m}$ core and coupled into a spectrometer with a charge coupled device.

\section{NUMERICAL RESULT}

While the light wave is diffracted from the photonic crystal structure with a triangular lattice, it should satisfy the phase match and energy conservation as follows [2]:

$$
\begin{gathered}
K_{d}=K_{i}+q_{1} K_{1}+q_{2} K_{2}, \quad q_{1,2}=0,1,2, \ldots \\
\omega_{d}=\omega_{i}
\end{gathered}
$$

where $K_{d}$ is a wave vector of the diffracted light wave; $K_{i}$ is an $x y$-plane wave vector of the incident light wave; $K_{1,2}$ are the Bragg wave vectors provided by the triangular lattice in the photonic crystal pattern; $q_{1}$ and $q_{2}$ are the orders of diffraction; $\omega_{d}$ is the frequency of the diffracted light wave, and $\omega_{i}$ is the frequency of the incident light. In addition, the dispersion curves or the photonic band structures for a triangular lattice with the transverse-electric (TE) polarization can be calculated by the PWEM as shown in Fig. 3. The Bragg condition and (14) are well satisfied, especially, at band edges in the photonic band structures due to the specific lattice constant, e.g., the $\Gamma 1, \mathrm{~K} 2$, and $\mathrm{M} 3$ band edges indicated by the black dashed circles in 


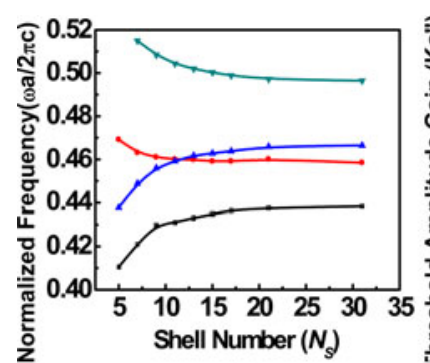

(a)

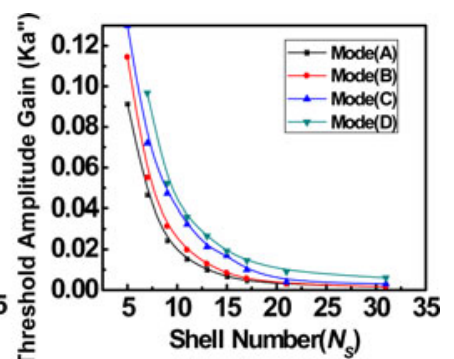

(b)
Fig. 4. (a) Normalized frequency versus the shell number of photonic crystals. (b) Threshold amplitude gain as a function of the shell number of cylinder layers with four resonant modes.

Fig. 3. These band edges, showing low group velocity and high density of states in photonic modes, are suitable for lasing to occur. Fig. 3 (right) shows the detailed band structure for each band edge. There are six bands at the $\Gamma 1$ band edge, three bands at $\mathrm{K} 2$ band edge, and four bands at the M3 band edge, resulting from different symmetric diffractions. As a result, the different band edges would exhibit different photonic characteristics. In addition, the special surface emission condition would occur at the band edges in 2-D PCSELs when the resonant modes are above the light line. For example, lasing modes at $\Gamma 1, \mathrm{~K} 2$, and M3 band edges in Fig. 3 should exhibit the surface out-coupling capability.

In this section, we discuss the results of numerical calculation based on the MSM. The resonant-mode frequencies, threshold gains, and field distributions in the reciprocal space at different band edges have been calculated.

\section{A. Influence of the Shell Number in Photonic Crystals}

To simplify the complex 3-D problems, the structural parameters used for this study are based on the effective index method [2]. The confinement factor of 0.563 for the photonic crystal layer and the effective index of 2.495 for the guided mode were calculated by the transfer matrix method [18], [19]. For the $\Gamma 1$ band edge, $\varepsilon_{a}$ of material $A$ (cylinder) was 1.87 and $\varepsilon_{b}$ of the background material $\mathrm{B}(\mathrm{GaN})$ was 2.65 , the filling factor of the cylinder was 0.25 , and the lattice constant was $180 \mathrm{~nm}$. There were seven partial waves in the expansion coefficient of the Bessel function $(n=-3$ to +3$)$. The determinant equation, i.e., $\operatorname{det}(M)=0$, calculated the normalized frequency and threshold amplitude gain. A size parameter $N_{s}$ shown in Fig. 2, represents the shell number of cylinder layers in the $\Gamma-\mathrm{M}$ direction. Fig. 4(a) shows the normalized frequency with respect to the shell number. Four resonant modes can be determined. It shows that when the shell number increases, the normalized frequency will approach to the steady value. The relation between the threshold gain and the shell number $N_{s}$ is shown in Fig. 4(b). The threshold gains of four resonant modes would exponentially decay, while increasing the shell number of photonic crystals, and the marginal influence of the shell number tends to converge after $N_{s}$ is larger than 21 in our simulation model. It indicates that the influence of PC shell would be saturated and threshold gains would approach to a constant since the lasing

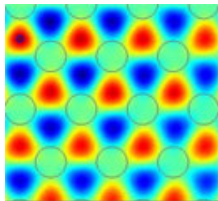

(a)

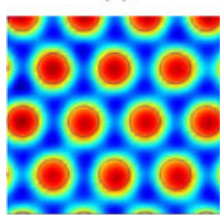

(c)

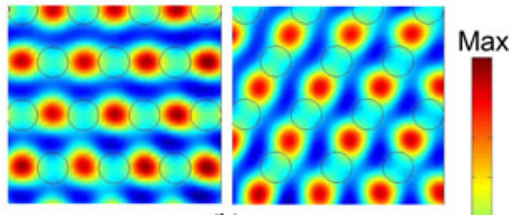

(b)

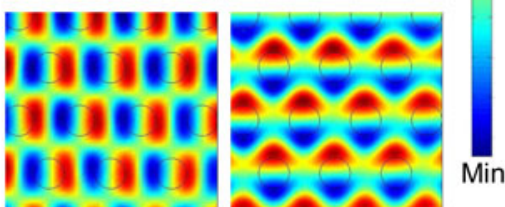

(d)
Fig. 5. Magnetic-field distributions of four resonant modes at the $\Gamma 1$ band edge for (a) mode A, (b) mode B, (c) mode C, and (d) mode D. Blue and red areas represent the positive and negative magnetic fields perpendicular to the plane, respectively. Black circles indicate the photonic crystal cylinders.

mechanism of PCSELs is provided by the DFB in a photonic crystal lattice and the boundary reflections around the photonic crystal structure. If there are only few shells of cylinders, the boundary reflections will seriously influence the laser threshold. As the shell number of photonic crystal increases, the feedback mechanism will be dominated by the photonic crystal diffraction, and thus, the lasing threshold converges. Simultaneously, the normalized lasing modes would approach the band-edge positions. These values are the steady-state solutions that match well to the results obtained in the PWEM and can be used in the following calculation. As a result, the shell number was set to be 21 in the following calculations.

Most of the previous GaN-based PCSELs were designed to operate at the $\Gamma 1$ band edge to form the surface emission condition [5]-[7] due to its strong coupling characteristics. As shown in Fig. 3, the band diagram around $\Gamma 1$ band edge indicates four associated resonant modes, termed as modes A, B, C, and D, respectively, from low to high normalized frequency. Modes $\mathrm{B}$ and D are doubly degenerate coupling waves. It can be distinguished into two pairs of doubly degenerate bands and two pairs of nondegenerate bands. These resonant modes can also be identified from the magnetic-field distributions, which are calculated by (10) and (11) by adding the internal and external magnetic-field distributions as shown in Fig. 5. Each resonant mode shows distinct magnetic-field distributions. The magnetic field distribution of mode $\mathrm{A}$ is divided into three positive and negative areas around each lattice point, referred as the hexapole mode. As for mode $\mathrm{B}$, there are two positive and negative areas around each lattice point, which is identified as the quadruple mode. Modes $\mathrm{C}$ and $\mathrm{D}$ are characterized by monopole and dipole modes, respectively. The normalized frequencies of modes A-D were calculated to be $0.4376,0.4601,0.4659$, and 0.4972 , respectively.

\section{B. Real-Space Resonant-Mode Patterns for K2 and M3 Band Edges}

Except for the $\Gamma 1$ band edge, there are other higher order band edges, such as K2 and M3, from the band diagram in Fig. 3. The resonant characteristics including mode patterns and threshold 


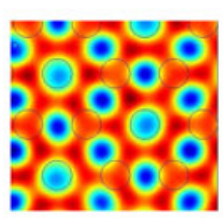

(a)

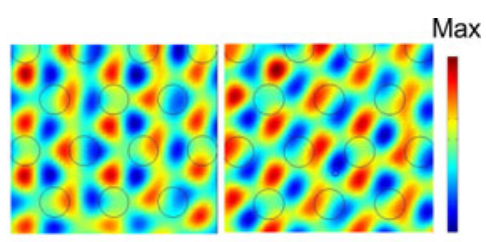

(b)
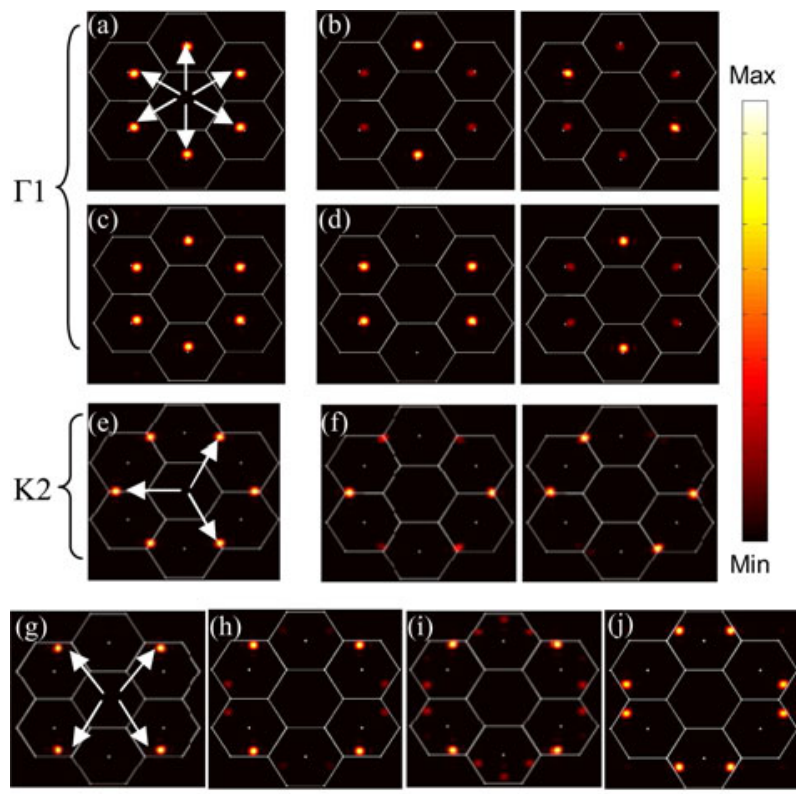

M3

gains of $\mathrm{K} 2$ and M3 band edges can also be investigated and discussed by using the MSM. Fig. 6 shows all of the mode patterns at $\mathrm{K} 2$ and $\mathrm{M} 3$ band edges. For the K2 band edge, $\varepsilon_{a}=$ 2 and $\varepsilon_{b}=2.63$ were used for materials A and $\mathrm{B}$, respectively. The filling factor of the cylinder was 0.26 and the lattice constant was $220 \mathrm{~nm}$. As shown in Fig. 3, the K2 band edge can exhibit two resonant modes. The normalized frequency of each resonant mode was calculated to be 0.52327 , and 0.55286 , respectively. In Fig. 6(a), the field distribution of the lower frequency mode $\mathrm{E}$ has three negative areas around each lattice point. Fig. 6(b) shows the higher frequency degenerate modes with two opposite dipoles around every lattice point. It should note that the field distribution would be changed due to the finite photonic crystal boundary. Similarly, for the M3 band edge, $\varepsilon_{a}=2.0$ and $\varepsilon_{b}$ $=2.63$ were used for materials $\mathrm{A}$ and for $\mathrm{B}$, respectively. The filling factor of the cylinder was 0.266 and the lattice constant was $247 \mathrm{~nm}$. Compared with the $\Gamma 1$ band edge, there also exist four modes termed as modes G, H, I, and J at the M3 band edge. Each mode pattern shows significant symmetric magnetic-field distributions in Fig. 6(c)-(f). Modes G-I shows three similar kinds of magnetic-field distributions in Fig. 6(d)-(f). In Fig. 6(f), mode $\mathrm{J}$ has a pair of positive and negative areas around each lattice point. The normalized frequencies corresponding to $\mathrm{G}$, $\mathrm{H}, \mathrm{I}$, and $\mathrm{J}$ modes were calculated to be $0.59348,0.60968$, 0.61571 , and 0.62408 , respectively.

\section{Reciprocal-Space Mode Patterns}

In order to investigate more features of different modes, it is useful to transfer the magnetic-field distributions in the real space into the reciprocal space or $k$ (wave number) space by using (6). Fig. 7 shows the magnetic fields in the reciprocal space and the white hexagonal lines represent the Brillouinzone boundaries for a triangular lattice. For the $\Gamma 1$ band edge, the field distribution of modes $\mathrm{A}$ and $\mathrm{C}$ locates at six zeniths of a hexagon in the second Brillouin zone, representing that these six diffraction directions strongly couple together to enhance the inplane laser oscillation. On the other hand, the reciprocal-space

Fig. 7. Calculated magnetic-field distributions of $\Gamma 1, \mathrm{~K} 2$, and $\mathrm{M} 3$ band edges in the reciprocal space. The white arrows indicate the diffraction direction for each band-edge points.

field distributions of modes $\mathrm{B}$ and $\mathrm{D}$ show two major diffraction directions, representing their specific laser oscillation directions. Since these diffraction patterns are in the $\Gamma$ points of six second Brillouin zone, these modes can be diffracted back to the $\Gamma$ point of the first Brillouin zone by the first-order diffraction. As a result, the output emission shall be normal to the surface. The same analysis can be applied to other band edges. The K2 bandedge mode emits the diffracted lights tilting an angle of $30^{\circ}$ off to the normal of the surface, which can be extracted from the reciprocal field patterns as shown in Fig. 7 (middle). Similarly, the M3 band edge mode emits several inclined diffracted lights. By observing the reciprocal field patterns shown in Fig. 7 (bottom), the tilted angles of these inclined diffracted lights can be calculated as $19.47^{\circ}, 35.26^{\circ}$, and $61.87^{\circ}$ off to the normal of the PC surface, respectively. These values are in good agreement with our previous experiment results [19].

\section{Threshold Gains for Different Band Edges}

Finally, the GaN-based PCSELs operated at different band edges were measured under the optical pumping system at room temperature. The gain peak of MQWs was at $410-420 \mathrm{~nm}$ with a linewidth of about $30 \mathrm{~nm}$. The lasing wavelengths for $\Gamma 1, \mathrm{~K} 2$, and M3 band-edge points are 416, 402, and $392 \mathrm{~nm}$, respectively. a/ $\lambda$ of the lasing threshold for $\Gamma 1, \mathrm{~K} 2$, and $\mathrm{M} 3$ band-edge points are 0.46, 0.54, 0.63, respectively. As shown in Fig. 8, clear threshold conditions are observed with the threshold power density of $3.7 \mathrm{~mJ} / \mathrm{cm}^{2}$ for the $\Gamma 1$ band edge, $6.3 \mathrm{~mJ} / \mathrm{cm}^{2}$ for the $\mathrm{K} 2$ band edge, and $8.1 \mathrm{~mJ} / \mathrm{cm}^{2}$ of the M3 band edge, showing a tendency of the increased threshold from low- to high-order bands. According to the simulation results, the threshold amplitude gains were $6.2 \times 10^{-3}, 5.9 \times 10^{-3}, 7.7 \times 10^{-3}$, and $7.3 \times 10^{-3}$ for 


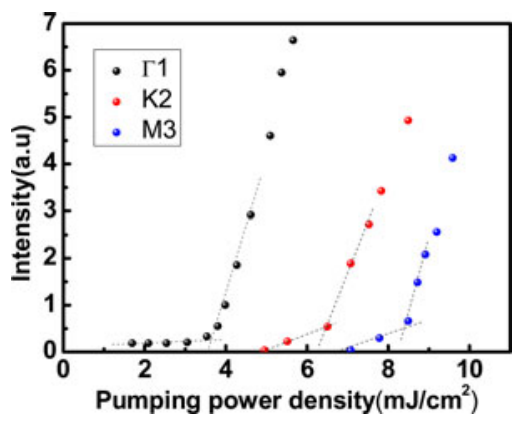

Fig. 8. Measured output intensity versus input excitation energy density from the GaN-based PCSELs for $\Gamma 1, \mathrm{~K} 2$, and $\mathrm{M} 3$ band edges.

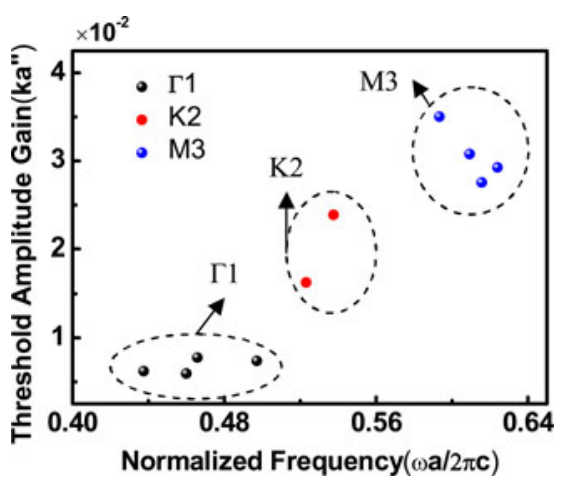

Fig. 9. Calculated threshold gains and normalized frequency for $\Gamma 1, \mathrm{~K} 2$, and M3 band edges of GaN-based PCSELs.

modes A, B, C, and D of the $\Gamma 1$ band edge, respectively. The different simulation result of the threshold amplitude gain comes from the variation distribution of field in a photonic crystal. It depends on the magnetic distribution field in the gain material. As shown in Fig. 6, modes A (hexagonal pole) and B (quadrant pole) have the more distributed field in the gain material than modes $\mathrm{C}$ (mono pole) and $\mathrm{D}$ (dipole mode); thus, modes $\mathrm{A}$ and $\mathrm{B}$ have the lower threshold gain than modes $\mathrm{C}$ and $\mathrm{D}$.

For the K2 band edge, the threshold amplitude gains were calculated to be $1.6 \times 10^{-2}$ and $2.4 \times 10^{-2}$. The lower normalized frequency mode has a relatively lower threshold gain. As for the M3 band edge, we obtained the threshold amplitude gains of $3.5 \times 10^{-2}, 3.0 \times 10^{-2}, 2.7 \times 10^{-2}$, and $2.9 \times 10^{-2}$. The K2 band edge has the same result as the $\Gamma 1$ band edge that the threshold gain of the hexagonal mode $\mathrm{E}$ is lower than the dipole mode $\mathrm{F}$ due to the less overlap of the magnetic field with the gain material. The same result can be obtained that the dipole mode $\mathrm{G}$ has the highest threshold amplitude gain at the M3 band edge. Fig. 9 shows the calculated threshold gains versus the normalized frequency for three band edges. The black dashed eclipses group three band-edge modes. Typically, modes at the $\Gamma 1$ band edge have the lowest threshold gain and modes at the M3 band edge have higher threshold gains than others. This can be schematically understood from the following. The white arrows in Fig. 7 indicate the length and directions of $\mathrm{k}$ vectors oscillating in the reciprocal space. The longer $\mathrm{k}$ vector means lower coupling coefficient due to the deviation from the Bragg vectors provided by the photonic crystal lattice [3], [16], [20]; thus, the $\Gamma 1$ has the lower threshold gain than $\mathrm{K} 2$ and $\mathrm{M} 3$ due to stronger coupling enhancement. Although the measured results could not be resolved into exact resonant modes for each band edge, the calculated results still show great agreement with the experimental results, indicating that the MSM could correctly predict the threshold gain in each band-edge mode and provide a fast design tool for the GaN-based PCSELs.

\section{CONCLUSION}

In this paper, we have employed the MSM to investigate the characteristics of different band-edge modes in GaN-based PCSELs with the triangular photonic crystal lattice. The different mode patterns in the real space can be calculated and classified for different band edges. In addition, the different mode patterns in the reciprocal space have been calculated and discussed. The diffraction spots in the reciprocal space could be used to identify the oscillation directions and output emission angles of each band-edge mode. Finally, the GaN-based PCSELs operated at $\Gamma 1, \mathrm{~K} 2$, and $\mathrm{M} 3$ band edges were measured and simulated by the MSM. The threshold condition at each band edges shows a clear tendency of the increased threshold from low- to highorder bands. The simulation results show good agreement with the experimental results. We believe that the presented results will be beneficial to the calculation of PCSEL devices and useful for the development of the novel PCSEL devices in the near future.

\section{ACKNOWLEDGMENT}

The authors would like to gratefully acknowledge Prof. P. G. Luan at National Central University, Zhongli, Taiwan, for fruitful suggestions, and Prof. S. C. Wang and H. C. Kuo at National Chiao Tung University, Hsinchu, Taiwan, for their technical support.

\section{REFERENCES}

[1] M. Meier, A. Mekis, A. Dodabalapur, A. Timko, R. E. Slusher, J. D. Joannopoulos, and O. Nalamasu, "Laser action from two-dimensional distributed feedback in photonic crystals," Appl. Phys. Lett., vol. 74, p. 7, 1999.

[2] M. Imada, A. Chutinan, S. Noda, and M. Mochizuki, "Multidirectionally distributed feedback photonic crystal lasers," Phys. Rev. B., vol. 65, p. 195306, 2002.

[3] I. Vurgaftman and J. Meyer, "Design optimization for high-brightness surface-emitting photonic-crystal distributed-feedback lasers," IEEE J. Quantum. Electron., vol. 39, no. 6, pp. 689-700, Jun. 2003.

[4] E. Matioli, E. Rangel, M. Iza, B. Fleury, N. Pfaff, J. Speck, E. Hu, and C. Weisbuch, "High extraction efficiency light-emitting diodes based on embedded air-gap photonic-crystals," Appl. Phys. Lett., vol. 96, p. 031108, 2008.

[5] H. Matsubara, S Yoshimoto, H. Saito, Y. Jianglin, Y. Tanaka, and S. Noda, "GaN photonic-crystal surface-emitting laser at blue-violet wavelengths," Science, vol. 319, pp. 445-447, 2008.

[6] T. C. Lu, S. W. Chen, L. F. Lin, T. T. Kao, C. C. Kao, P. Yu, H. C. Kuo, S. C. Wang, and S. H. Fan, "GaN-based two-dimensional surface-emitting photonic crystal lasers with AlN/GaN distributed Bragg reflector," Appl. Phys. Lett., vol. 92, p. 011129, 2008.

[7] S. Kawashima, T. Kawashima, Y. Nagatomo, Y. Hori, H. Iwase, T. Uchida, K. Hoshino, A. Numata, and M. Uchida, "GaN-based surface-emitting laser with two-dimensional photonic crystal acting as distributed-feedback grating and optical cladding," Appl. Phys. Lett., vol. 97, p. 251112, 2010.

[8] M. Kim, C. S. Kim, W. W. Bewley, J. R. Lindle, C. L. Canedy, I. Vurgaftman, and J. R. Meyer, "Surface-emitting photonic-crystal distributed- 
feedback laser for the midinfrared," Appl. Phys. Lett., vol. 88, p. 191105, 2006.

[9] L. Sirigu, R. Terazzi, I. Amanti, M. Giovannini, J. Faist, A. Dunbar, and R. Houdré, "Terahertz quantum cascade lasers based on two-dimensional photonic crystal resonators," Opt. Exp., vol. 16, pp. 5206-5217, 2008.

[10] K. Sakoda, K. Ohtaka, and T. Ueta, "Low-threshold laser oscillation due to group-velocity anomaly peculiar to two- and three-dimensional photonic crystals," Opt. Exp., vol. 4, pp. 481-489, 1999.

[11] K. Sakai, J. Yue, and S. Noda, "Coupled-wave model for triangular-lattice photonic crystal with transverse electric polarization," Opts Exp., vol. 16, pp. 6033-6040, 2008.

[12] K. Sakai, E. Miyai, and S. Noda, "Coupled-wave theory for square-lattice photonic crystal lasers with TE polarization," IEEE J. Quantum Electron., vol. 46, no. 5, pp. 788-795, May 2010.

[13] Y. H. Lee, H. Y. Ryu, and M. Notomi, "Finite-difference time-domain investigation of band-edge resonant modes in finite-size two-dimensional photonic crystal slab," Phys. Rev. B., vol. 68, p. 045209, 2003.

[14] S. Nojima, "Theoretical analysis of feedback mechanisms of twodimensional finite-sized photonic-crystal lasers," J. Appl. Phys., vol. 98, p. 043102, 2005.

[15] S. Nojima, "Optical-gain enhancement in two-dimensional active photonic crystals," J. Appl. Phys., vol. 90, pp. 545-551, 2001.

[16] S. W. Chen, T. C. Lu, and T. T. Kao, "Study of GaN-based photonic crystal surface-emitting lasers (PCSELs) with AlN/GaN distributed Bragg reflectors," IEEE J.Sel.Topics Quantum. Electron., vol. 15, no. 3, pp. 885891, May/Jun. 2009.

[17] Y. Y. Chen and Z. Ye, "Propagation inhibition and wave localization in a two-dimensional random liquid medium," Phys. Rev. E., vol. 65, p. 056612, 2002.

[18] P. S. Weng, T. T. Wu, T. C. Lu, and S. C. Wang, "Threshold gain analysis in GaN-based photonic crystal surface emitting laser," Opt. Lett., vol. 36, pp. 1908-1910, 2011.

[19] S. W. Chen, T. C. Lu, Y. J. Hou, T. C. Liu, H. C. Kuo, and S. C. Wang, "Lasing characteristics at different band edges in GaN photonic crystal surface emitting lasers," Appl. Phys Lett., vol. 96, p. 071108, 2010.

[20] K. Sakai, J. Yue, and S. Noda, "Coupled-wave model for triangular-lattice photonic crystal with transverse electric polarization," Opt. Exp., vol. 16, no. 9, pp. 6033-6040, 2008.

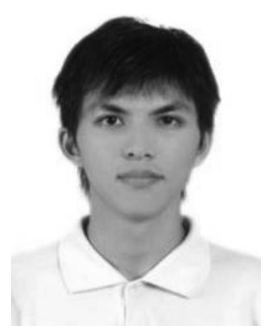

Peng-Hsiang Weng received the B.S. degree in physics from the National Tsing-Hua University, Hsinchu, Taiwan, in 2008, and the M.S. degree in electro-optical engineering from the National Chiao Tung University (NCTU), Hsinchu, in 2010.

In 2008, he joined the Semiconductor Laser Technology Laboratory, NCTU, under the supervision of Prof. T.-C. Lu, Prof. H.-C. Kuo, and Prof. S.-C. Wang. $\mathrm{He}$ is currently a Full Time Research Assistant in the NCTU. His current research interests include GaN PCSEL, defect PCSEL, and simulation method pro-

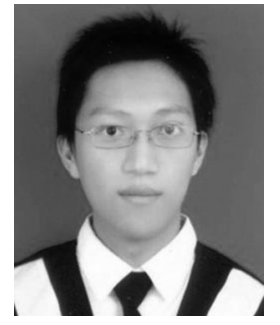

Tzeng-Tsong $\mathrm{Wu}$ received the B.S. degree in electrical engineering, in 2007, and the M.S. degree in optoelectronic sciences, in 2009, both from the $\mathrm{Na}$ tional Taiwan Ocean University (NTOU), Keelung, Taiwan. He is currently working toward the Ph.D. degree at the Department of Photonics and Institute of Electro-Optical Engineering, National Chiao Tung University (NCTU), Hsinchu, Taiwan.

$\mathrm{He}$ joined the Semiconductor Laser Technology Laboratory, NCTU, in 2009, where he was engaged in research on III-V semiconductor materials for semiconductor lasers under the supervision of Prof. T.-C. Lu and Prof. S.-C. Wang. His recent research interests include GaN-based vertical cavity SEL (VCSEL) and PCSEL.

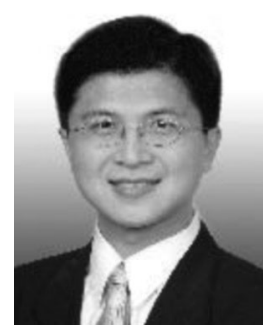

Tien-Chang Lu (M'07) received the B.S. degree in electrical engineering from the National Taiwan University, Taipei, Taiwan, in 1995, the M.S. degree in electrical engineering from the University of Southern California, Los Angeles, in 1998, and the Ph.D. degree in electrical engineering and computer science from the National Chiao Tung University, Hsinchu, Taiwan, in 2004.

He was a Manager at the Epitaxy Department Union Optronics Corporation, Yangmei, Taiwan, in 2004. Since August 2005, he has been a Faculty Member in the Department of Photonics, National Chiao Tung University Hsinchu, Taiwan. His research interests include the design, epitaxial growth, process, and characterization of optoelectronic devices. He has been engaged in the lowpressure MOCVD epitaxial technique associated with various material systems, as well as the corresponding process skills. His research interests also include the structure design and simulations for optoelectronic devices using computeraided software. He has authored and coauthored more than 150 international journal papers.

Dr. Lu is a recipient of The Exploration Research Award of Pan Wen Yuan Foundation 2007, Excellent Young Electronic Engineer Award 2008, and Young Optical Engineering Award 2010. 